\title{
Fracture Resistance of Teeth with Truss Endodontic Access: An In Vitro Study and Literature Review
}

\author{
Mohmed Isaqali Karobari ${ }^{1, \odot ~ A h m a d ~ F a r e e z ~ A b d u l ~ A z i z ~}{ }^{1} \quad$ Saleem D. Makandar ${ }^{1,2}$ \\ Nik Rozainah Nik Abdul Ghani ${ }^{1,2}$ Mohamad Syahrizal Halim ${ }^{1,2, \odot}$ Tahir Yusuf Noorani ${ }^{1,2, \odot}$ \\ ${ }^{1}$ Conservative Dentistry Unit, School of Dental Sciences, Universiti \\ Address for correspondence Tahir Yusuf Noorani, DDS, MResDent, \\ Sains Malaysia, Health campus, Kota Bharu, Kelantan, Malaysia \\ ${ }^{2}$ Conservative Dentistry Unit, Hospital Universiti Sains Malaysia, \\ Health campus, Kota Bharu, Kelantan, Malaysia \\ FRACDS, Conservative Dentistry Unit, School of Dental Sciences, \\ Universiti Sains Malaysia, Health campus, 16150 Kubang Kerian, \\ Kota Bharu, Kelantan, Malaysia (e-mail: dentaltahir@yahoo.com).
}

Eur J Gen Dent:2021;10:44-49

\author{
Abstract \\ Keywords \\ - conservative access \\ cavity \\ - dental pulp \\ - endodontics \\ - endodontic access \\ cavity \\ - traditional access \\ cavity
}

Objective This article evaluates the fracture resistance of teeth with different endodontic access cavities and conducts literature review on the effects of truss endodontic cavity.

Material and Methods Eighty human mandibular first molars were assigned randomly to four groups ( $n=20)$; group 1: truss access cavity (TrussAC); group 2: conservative access cavity (ConsAC); group 3: traditional access cavity (TradAC); and group 4: served as control (no access). Accesses were prepared in groups 1 to 3 , respectively. Endodontic treatment was performed on all accessed teeth and composite restoration placed. Teeth in all groups were loaded until fracture using a mechanical testing machine. The maximum load at fracture and patterns of fracture were recorded. Additionally, a literature search was performed on studies related to TrussAC and its importance.

Statistical Analysis Fracture strengths were compared using one-way analysis of variance complemented by Bonferroni test. Fracture patterns were analyzed using chi-square test.

Results TradAC showed significantly lower mean load at fracture than TrussAC, ConsAC, and intact teeth. Fracture resistance of teeth with TrussAC and ConsAC was not significantly different. All three test groups showed significantly more frequent unrestorable fractures. The literature review revealed that TrussAC did not improve the fracture strength significantly; however, pulp chamber debridement was significantly impaired.

Conclusion TrussAC improved the fracture strength of endodontically treated teeth, but not significantly as compared to ConsAC. Restorable fractures were more in intact teeth compared with all prepared ones.
DOI https://doi.org/

$10.1055 / \mathrm{s}-0041-1732780$

ISSN 2320-4753 (c) 2021. European Journal of General Dentistry.

This is an open access article published by Thieme under the terms of the Creative Commons Attribution-NonDerivative-NonCommercial-License, permitting copying and reproduction so long as the original work is given appropriate credit. Contents may not be used for commercial purposes, or adapted, remixed, transformed or built upon. (https://creativecommons.org/licenses/by-nc-nd/4.0/).

Thieme Medical and Scientific Publishers Pvt. Ltd. A-12, 2nd Floor, Sector 2, Noida-201301 UP, India 


\section{Introduction}

Endodontic treatment is the standard care for a nonvital or endodontically involved tooth. However, endodontically treated teeth are weakened and generally are more prone to fracture. ${ }^{1,2}$ Loss of tooth structure, either due to caries or endodontic procedures, including the loss due to endodontic access cavity preparation is considered a major risk factor for fractures. ${ }^{3}$ Minimally invasive treatments are trending in modern dentistry. Thus, in endodontics, the concept of contracted access cavity was born because a tooth's susceptibility to fracture was found to be proportional to the amount of tooth structure removed. ${ }^{4}$

Traditional access cavity (TradAC) designs for all types of teeth have been the same since decades with only slight alterations. TradAC involves removal of tooth structure to allow access to the canals, facilitating complete cleaning, shaping, and obturation, while preventing procedural complications. However, excessive tooth structure is removed in a TradAC. Moreover, this affects the tooth's strength to resist fracture when subjected to loading in function. Thus, with a reduced and properly designed endodontic access cavity, the prognosis of a tooth that undergoes endodontic treatment can be improved. ${ }^{5,6}$

Meanwhile, in a minimally invasive procedure called conservative access cavity (ConsAC), tooth structure including the peri-cervical dentine can be preserved. ${ }^{4}$ There are no particular rules to prepare a ConsAC. The main aim is to conserve maximum tooth structure while locating all the canal orifices.

Truss access cavity (TrussAC) on the other hand has been recently introduced to further preserve the tooth structure. ${ }^{7}$ Cone beam computed tomography information of the tooth together with magnification is used to prepare strategically located truss access. ${ }^{8}$ These accesses are placed directly above the respective root canals, and through these holes, cleaning, shaping, and obturation is performed. Hence, complete root canal treatment (RCT) is performed either through existing restorative cavities or by creating small entries leaving lateral pulp horns and the rest of the pulp chamber unroofed. ${ }^{7}$ Many studies have been performed to determine fracture resistance of teeth with ConsAC $C^{1,4,9,10}$; however, there is limited and conflicting evidence available on the fracture resistance of teeth with TrussAC. ${ }^{11}$ Thus, this study aimed to determine the effect of three different access cavity designs on fracture resistance of endodontically treated mandibular molars.

\section{Materials and Methods}

In this in vitro experimental study, 80 recently extracted first permanent mandibular molars were obtained from dental clinics of School of Dental Sciences, Universiti Sains Malaysia (USM), Kota Bharu, Kelantan, Malaysia. Teeth were extracted due to periodontal conditions. The sample size calculation was done using input parameters, effect size 0.04 with $80 \%$ power, and $\alpha$ of 0.05 as per a previous study ${ }^{7}$ to obtain a sample size of 20 in each group. Ethics Committee for Human Research, USM (Ref. USM/JEPeM/17040221) approved this study.
All teeth were inspected for the absence of previous restorations, fractures, or abrasion under a microscope (Leica Microsystem Imaging Solutions, Cambridge, United Kingdom) at a 20× magnification. Soft tissue remnants and calculus were cleaned off the surfaces of the selected teeth with a hand curette. The teeth were then disinfected by soaking for 10 minutes in sodium hypochlorite ( $\mathrm{NaOCl}$ ) (5.25\%; Clorox Inc, Oakland, United States), followed by rinsing with distilled water. Subsequently, the mesiodistal and buccolingual dimensions of the teeth were measured using a digital caliper at the cervical margin. Teeth included in the study had mesiodistal and buccolingual dimension of $10.61 \mathrm{~mm}$ $( \pm 0.45 \mathrm{~mm})$ and $10.25 \mathrm{~mm}( \pm 0.45 \mathrm{~mm})$, respectively. The mean mesiodistal and buccolingual pulp chamber distance were 3.76 and $4.39 \mathrm{~mm}$, respectively, measured from preoperative computed tomographic images. Teeth were stored in $10 \%$ formalin solution until use.

Eventually, 20 teeth were allocated to each group as follows:

Group 1: Teeth with TrussAC prepared.

Group 2: Teeth with ConsAC prepared.

Group 3: Teeth with TradAC prepared.

Group 4: Control group; intact teeth.

TrussAC, ConsAC, and TradAC were prepared on all teeth respectively using an endo access bur size 2, $21 \mathrm{~mm}$ (Dentsply Maillefer, United States) mounted on a high speed handpiece, water spray was used as coolant. In group 1, truss accesses were prepared above the mesial and distal roots utilizing preoperative computed tomographic images as a guide, ensuring the canal orifices could be located, leaving a truss in between the mesial and distal cavities. Hence, a small access and tiny entries left the pulp chamber roof and lateral pulp horns intact $^{7,12}$ ( - Fig. 1A). In group 2, the ConsAC preparation was initiated at the central fossa (mesial quarter) and extended distally and apically until all canals were located. Peri-cervical dentin removal mesiodistally, buccolingually, and circumferentially was minimized ensuring pulp chamber roof was partly maintained and all root canal orifices could be localized visually from the same angulation ${ }^{4}$ (-Fig. 1B). TradAC preparation was performed on teeth in group 3 following the TradAC principles as reported in earlier studies ${ }^{1,7}$ ( - Fig. 1C). RCT was then done in all access cavity groups. A K-file size 10 (FlexOFiles; Dentsply Maillefer, Switzerland) was used to check canal patency. Working length was determined by introducing a file till it was visible beyond the apical foramen, $1 \mathrm{~mm}$ was then subtracted and determined as the working length. Instrumentation was then performed using NiTi rotary files (S5 Sendoline, Sweden) until final apical size 30, 0.06 taper following manufacturers' instructions. Irrigation with $2.5 \% \mathrm{NaOCl}$ solution (Lenntech, Delfgauw, Netherlands) was performed during instrumentation. Finally, $5 \mathrm{~mL}$ of $17 \%$ ethylenediaminetetraacetic acid solution (Promega Corporation, Wisconsin, United States) followed by $10 \mathrm{~mL}$ of normal saline solution (RMBIO, Missoula, Montana, United States) was used. Obturation was subsequently performed using gutta-percha (Dentsply Maillefer, Switzerland) and AH Plus sealer (Dentsply Maillefer, Switzerland) using warm vertical condensation technique. Throughout the endodontic 


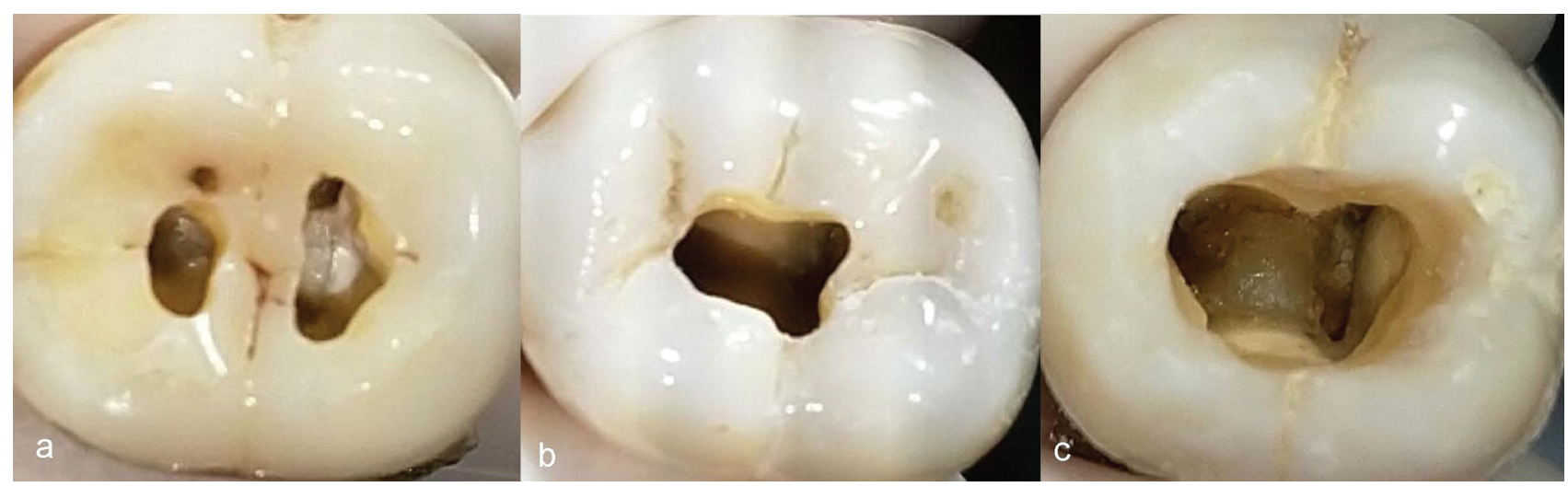

Fig. 1 Access cavity designs for each group: (A) Truss access cavity; (B) Conservative access cavity; (C) Traditional access cavity.

procedure the teeth were handled using a moistened gauze to avoid dehydration of the samples.

After completion of endodontic treatment, the access cavities were cleaned using a spoon excavator followed by a moistened cotton pellet to remove all the debris. Phosphoric acid 37\% (Magic Acid Coltene, Brazil) was used for etching (enamel for 30 and dentine for 15 seconds). Subsequently, the cavity was rinsed with water/air spray for 30 seconds, and air-dried gently to avoid desiccation. An adhesive (OptiBond S; Kerr Corporation, California, United States) was then applied, thinned by gentle air blowing, and polymerized using a light-emitting diode for 10 seconds. Finally, composite resin (Zmack Comp, Italy) was placed incrementally ( $2 \mathrm{~mm}$ increments) to restore the access cavities and each increment polymerized for 40 seconds following the manufacturers' instructions. Subsequently, composite restoration was polished using a composite polishing kit (Shofu, United States).

All teeth were then mounted with the roots $2 \mathrm{~mm}$ below the level of cementoenamel junction embedded in self-cured resin. Silicon impression material was used to simulate the periodontal ligament ((Panasil Light Body; Kettenbach GmbH \& Co KG, Eschenburg, Germany). Next, the specimens were mounted in a universal testing machine (AGS-X, Shimadzu, Japan). The teeth were loaded using a spherical steel tip of $3 \mathrm{~mm}$ diameter at a speed of $1 \mathrm{~mm} / \mathrm{min}$. Forces were then applied vertically on their central fossa, parallel to the tooth's long axis. The load at which the teeth fractured was recorded in Newton (N) as indicated by the load testing machine software. ${ }^{13}$ Fracture levels were then determined by examining the specimens using a stereomicroscope (Stereo Discovery, Zeiss, Germany). The following was used to classify fracture patterns:

Restorable: Fracture above the bone simulation level (acrylic resin).

Unrestorable: Fracture extending below the bone simulation level.

Normality of distribution was determined using the Kolmogorov-Smirnov test. One-way analysis of variance complemented with Bonferroni test was used for data analysis. Chi-square test was used to analyze the types of fractures. Significance level was set at $p=0.05$.

A literature search was then performed on January 6 , 2020 using PubMed and Google Scholar to find relevant articles using keywords, "Truss access cavity preparation," "Fracture strength with truss access cavity," "Truss access approach," and "Influence of truss access on fracture strength"; obtained literature was reviewed and summarized.

\section{Results}

The highest mean fracture resistance was found in group 4, followed by group 1 , group 2 , and group 3 demonstrating the lowest mean fracture resistance ( $\mathbf{- T a b l e ~ 1 )}$ ). Besides, pairwise

Table 1 Fracture strength $(\mathrm{N})$ analysis with comparison among groups using one-way ANOVA complemented with Bonferroni test

\begin{tabular}{|c|c|c|c|c|c|c|}
\hline \multirow[t]{2}{*}{$\begin{array}{l}\text { Groups } \\
(n=20)\end{array}$} & \multirow[t]{2}{*}{ Mean } & \multirow[t]{2}{*}{ SD } & \multirow{2}{*}{$\begin{array}{l}\text { One-way } \\
\text { ANOVA } \\
\text { test results } \\
p \text {-value }\end{array}$} & \multicolumn{3}{|c|}{$\begin{array}{c}\text { Bonferroni test } \\
\text { test results } \\
p \text {-value }\end{array}$} \\
\hline & & & & ConsAC & TradAC & Control \\
\hline TrussAC & 2068.07 & 357.39 & \multirow[t]{4}{*}{$<0.001^{\mathrm{a}}$} & 0.524 & $<0.001^{\mathrm{a}}$ & $0.042^{\mathrm{a}}$ \\
\hline ConsAC & 1954.41 & 224.30 & & & $<0.001^{\mathrm{a}}$ & $0.007^{a}$ \\
\hline TradAC & 1254.77 & 122.82 & & & & $<0.001$ \\
\hline Control & 2435.66 & 237.67 & & & & \\
\hline
\end{tabular}

Abbreviations: ANOVA, analysis of variance; ConsAC, conservative access cavity; SD, standard deviation; TradAC, traditional access cavity; TrussAC, truss access cavity.

aStatistically significant $(p<0.05)$. 
Table 2 List of studies and cases that determined the effects TrussAC

\begin{tabular}{|c|c|c|c|}
\hline Author (year) & Purpose of study & Methodology & Outcomes \\
\hline $\begin{array}{l}\text { Corsentino et } \mathrm{al}^{7} \\
(2018)\end{array}$ & $\begin{array}{l}\text { Assess influence of access cav- } \\
\text { ity design (TradAC, ConsAC, } \\
\text { TrussAC) and remaining tooth } \\
\text { substance on fracture strength }\end{array}$ & $\begin{array}{l}\text { Sound mandibular molar teeth were } \\
\text { selected. After access cavity preparation, } \\
\text { and removal of no, one, or two marginal } \\
\text { walls, teeth were endodontically treated } \\
\text { and restored. The specimens were then } \\
\text { loaded to fracture in a universal loading } \\
\text { machine }\end{array}$ & $\begin{array}{l}\text { TrussAC and ConsAC do not } \\
\text { increase the fracture strength of } \\
\text { endo treated teeth, rather the } \\
\text { loss of mesial and distal ridges } \\
\text { reduced fracture strength of teeth } \\
\text { significantly }\end{array}$ \\
\hline $\begin{array}{l}\text { Özyürek et al }{ }^{14} \\
\text { (2018) }\end{array}$ & $\begin{array}{l}\text { Assess the effects of endodon- } \\
\text { tic access cavity preparation } \\
\text { design (TradAC, TrussAC) } \\
\text { and different restorative } \\
\text { base material on the fracture } \\
\text { strength }\end{array}$ & $\begin{array}{l}\text { Intact mandibular molar teeth were } \\
\text { randomly assigned to TradAC or TrussAC } \\
\text { group (with one marginal wall missing), } \\
\text { restored with either SDR or EverX pos- } \\
\text { terior as base material. Samples loaded } \\
\text { after restoration until fracture }\end{array}$ & $\begin{array}{l}\text { TrussAC did not increase the frac- } \\
\text { ture strength of teeth. No difference } \\
\text { in the fracture strength between } \\
\text { teeth with TradAC or TrussAC when } \\
\text { the same base material was used }\end{array}$ \\
\hline $\begin{array}{l}\text { Neelakantan et al }{ }^{12} \\
(2018)\end{array}$ & $\begin{array}{l}\text { Assess the influence of using } \\
\text { TradAC and TrussAC access } \\
\text { cavity design on the efficacy to } \\
\text { debride the pulp space }\end{array}$ & $\begin{array}{l}\text { Intact mandibular molar teeth randomly } \\
\text { assigned to TradAC or TrussAC group, } \\
\text { endodontically treated and evaluated } \\
\text { histologically after sectioning to deter- } \\
\text { mine remaining pulp tissue }\end{array}$ & $\begin{array}{l}\text { The amount of pulp tissue remnants } \\
\text { was significantly lower in the pulp } \\
\text { chamber of TradAC group }\end{array}$ \\
\hline $\begin{array}{l}\text { Abou-Elnaga et al }{ }^{15} \\
\text { (2019) }\end{array}$ & $\begin{array}{l}\text { Assess the influence of TradAC, } \\
\text { TrussAC, and ATR on fracture } \\
\text { resistance }\end{array}$ & $\begin{array}{l}\text { Intact mandibular } 1 \text { st molars were } \\
\text { randomly assigned to TradAC, TrussAC, } \\
\text { and ATR. After access cavity preparation, } \\
\text { and removal of both marginal walls, } \\
\text { teeth were endodontically treated and } \\
\text { restored. The specimens were then } \\
\text { loaded to fracture }\end{array}$ & $\begin{array}{l}\text { The fracture resistance was not } \\
\text { significantly different between the } \\
\text { groups }\end{array}$ \\
\hline $\begin{array}{l}\text { Auswin and } \\
\text { Ramesh }^{16}(2017)\end{array}$ & $\begin{array}{l}\text { A case report on TrussAC new } \\
\text { conservative approach }\end{array}$ & $\begin{array}{l}\text { Right first molar root canal treatment } \\
\text { using TrussAC preparation }\end{array}$ & $\begin{array}{l}\text { This minimal invasive approach in } \\
\text { access opening prevents unneces- } \\
\text { sary tooth structure removal }\end{array}$ \\
\hline $\begin{array}{l}\text { Mooktiar et al }{ }^{17} \\
(2019)\end{array}$ & $\begin{array}{l}\text { A TrussAC preparation case } \\
\text { series }\end{array}$ & $\begin{array}{l}\text { Three permanent teeth with an indi- } \\
\text { cation for endodontic treatment were } \\
\text { treated via the Truss AC design }\end{array}$ & $\begin{array}{l}\text { This minimal invasive approach in } \\
\text { access opening avoids the need for } \\
\text { conventionally placed crowns }\end{array}$ \\
\hline
\end{tabular}

Abbreviations: ATR, artificial truss restoration; ConsAC, conservative access cavity; SDR, smart dentine replacement; TradAC, traditional access cavity; TrussAC, truss access cavity.

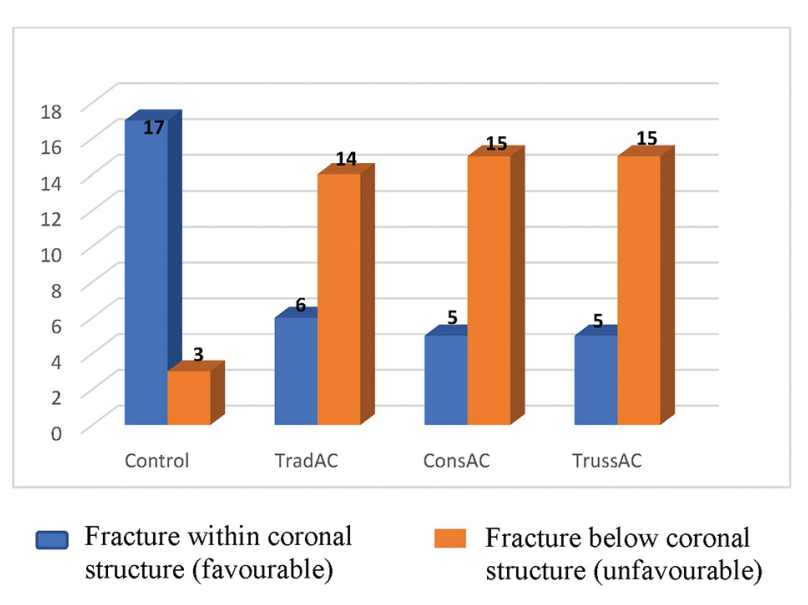

Fig. 2 Bar chart showing the fracture pattern among the four groups.

comparison of fracture resistance between groups showed significant difference between all groups, TrussAC and TradAC $(p<0.001)$, TrussAC and control $(p=0.042)$, ConsAC and TradAC $(p<0.001)$, ConsAC and control $(p=0.007)$, and TradAC and control $(p<0.001)$ except between TrussAC and ConsAC $(p=0.524)$.

In intact teeth (control group), a significantly higher number of restorable fractures were noted $(p=0.031)$. However, in all test groups, unrestorable fractures were significantly higher than the restorable ones $(p=0.028)$. This data are illustrated in -Fig. 2 . No correlation between the type of cavity and the favorability of fracture was found $(p=0.521$ ).

\section{Literature Review}

- Table 2 summarizes the list of studies that assessed the influence of TrussAC. A study was performed to determine whether the design of the endodontic access cavity (TradAC, ConsAC, TrussAC) and the amount of remaining tooth structure had any influence on the fracture strength of mandibular molars. It was found that loss of marginal ridges significantly affected the fracture strength rather than the endodontic cavity design itself. When the marginal ridges were intact, the teeth accessed with TrussAC, ConsAC, or TradAC had no significant difference in their fracture strength. ${ }^{7}$ Another similar study aimed to determine if different access cavity designs (TradAC and TrussAC) and use of different restorative base materials influenced the fracture strength of mandibular molars. It was found that the fracture strength did not increase with TrussAC. Furthermore, the fracture strength of teeth with TradAC or TrussAC was not significantly different when the same base material was used..$^{14}$ Another study was performed to assess the influence of using TradAC and 
TrussAC access cavity design on the efficacy to debride the pulp chamber and mesial root canal systems of mandibular molars. The authors concluded that pulp tissue remnants were seen in both TradAC and TrussAC groups; however, the amount of pulp tissue remnants were significantly lower in the pulp chamber of the TradAC group. ${ }^{12}$ Furthermore, a recent study performed on endodontically treated mandibular first molars compared the fracture resistance of teeth with TradAC, TrussAC, and artificial truss restoration. The fracture resistance was not significantly different between the groups. ${ }^{15}$ Only one case report and one case series has been published on TrussAC design. However, no posttreatment follow-up is presented. ${ }^{16,17}$ Furthermore, posttreatment radiographic evaluation is not satisfactorily presented, and the methodology was also not adequately explained. ${ }^{16}$ Besides, both these reports did not use three-dimensional imaging to plan orifice-directed access cavities (TrussAC) which might lead to failure of detecting additional/accessory root canals.

\section{Discussion}

In this study, the fracture resistance of sound teeth and teeth accessed with TrussAC, ConsAC, and TradAC were compared. Endodontically treated teeth fracture most commonly due to loss of tooth structure, ${ }^{3}$ and following TradAC principles for endodontic access cavity preparation is one of the most important causes of tooth structure loss. ${ }^{18}$

In an attempt to reduce the risk of fracture in endodontically treated teeth, ConsAC and TrussAC were introduced..$^{10}$ However, to the best of our knowledge, only one previous study has compared the fracture resistance of teeth accessed with TrussAC and ConsAC. ${ }^{7}$ Thus, the present study tested the effect of TrussAC, ConsAC, and TradAC on the fracture strength of teeth treated endodontically. In endodontically treated posterior teeth, vertical fractures most commonly occur in mandibular molars, ${ }^{7,19}$ thus they were selected in this study. Furthermore, to enable loading test and to simulate procedures performed clinically, composite resin was used to restore the endodontic access cavities. It has been observed that fracture strength is reinstated by nearly $72 \%$ after endodontic access cavities are restored. ${ }^{3}$

All preparation of the specimen was performed by one expert operator to eliminate differences in results caused by a difference in skills of the operator. In this study, the fracture strength of the TradAC group was found to be the lowest in comparison to the control, TrussAC, and ConsAC groups. Likewise, a previous study comparing the fracture resistance of teeth with TradAC and ConsAC showed similar results. ${ }^{1}$ When cavities are prepared with TradAC principles, the cavity floor is also the pulp chamber floor. ${ }^{7}$ Since, the centrally located tooth structure in mandibular molars is subjected to heavy masticatory loads, in ConsAC, preserving a part of the pulp chamber roof helps in distributing the occlusal forces, thereby reducing the amount of forces reaching the pulpal chamber floor. ${ }^{10}$ An additional aim in conservative access is to preserve the peri-cervical dentin, which is very important for the lifetime and the optimal function of teeth. However, studies that compared the fracture resistance of teeth accessed with ConsAC and TradAC found no significant difference. ${ }^{7,9,14}$ Furthermore, Abou-Elnaga et al ${ }^{15}$ reported that in teeth prepared with TradAC and TrussAC, fracture resistance did not differ significantly. Contrasting findings among studies comparing TradAC, ConsAC, and/or TrussAC could be due to difference in the type of teeth used, methodology of the study, and type of restorative material used.

Additionally, in this study, fracture resistance of teeth accessed with ConsAC and TrussAC did not differ significantly. These results are in line with other studies that tested the fracture resistance of teeth accessed with TrussAC. ${ }^{7}$ However, fracture resistance of teeth with TradAC was significantly lower than those with TrussAC in this study. This result is in line with a recent study that compared the fracture resistance of teeth with TrussAC and TradAC with and without artificial aging. ${ }^{20}$ The authors concluded that TrussAC did not reduce the fracture strength as compared with sound teeth, nevertheless, when subjected to thermal stress the fracture strength of teeth accessed with both TrussAC and TradAC was significantly reduced. ${ }^{20}$ Besides, the efficacy of the entire root canal therapy can be influenced by a reduced access cavity. ${ }^{10}$ TrussAC can particularly affect the ability of detecting root canals and influence complete removal of debris and pulp tissue remnants. ${ }^{12}$

Moreover, looking at the pattern of fractures in intact teeth, unrestorable ones were significantly lower than restorable ones. On the contrary, in TradAC, ConsAC, and TrussAC groups, restorable ones were significantly lower than unrestorable ones. Similarly, previous studies also reported a higher number of unrestorable fractures in accessed teeth, irrespective of the access cavity design type used. ${ }^{1}$ Extended preparation in TradAC design greatly reduces the amount of sound dentine, ${ }^{21}$ thus increasing the tooth deformability resulting in fractures that are unfavorable to restore. Although ConsAC and TrussAC preserve more peri-cervical dentin and pulpal chamber roof, there is still a decrease in the fracture strength due to loss of tooth structure, leading to more unfavorable fractures. ${ }^{3}$

The limitations of this study include the use of static instead of dynamic force to determine the fracture strength. Additionally, the $\mathrm{pH}$, temperature, and other intraoral factors were not simulated; hence, results should be extrapolated to the clinical situation cautiously.

\section{Conclusion}

Considering the limitations, it is concluded that, lower fracture strength was observed in teeth with TradAC (group 3) as compared with TrussAC (group 1) or ConsAC (group 2). Furthermore, when accessed using TrussAC, the fracture strength was not greater than in teeth with ConsAC. Moreover, unrestorable fractures were more than restorable ones in all accessed teeth.

The literature review showed that accessing teeth with TrussAC had no significant effect on the fracture strength, rather the fracture strength was significantly reduced with the loss of one or more marginal ridges. Furthermore, Truss AC resulted in a higher amount of pulp tissues remnants in the 
pulp chamber which may lead to failure of endodontic treatment. These conclusions are based solely on in vitro studies and clinical translation may not be completely pertinent.

\section{Authors' Contributions}

M.I.K. contributed in preparation of the manuscript. A.F.A.A. conducted the study as part of the DDS program. S.D.M. and N.R.N.A.G. contributed in supervision of the project and proof reading of the manuscript. M.S.H. contributed in supervision of the project, design of the project, and proof reading of the manuscript. T.Y.N. contributed in concept, design of the study, obtained funding, supervision, and proof reading of the manuscript.

\section{Funding}

Support from Universiti Sains Malaysia, Short Term Grant (304/PPSG/6315195 \& 304/PPSG/6316456) and management of Hospital Universiti Sains Malaysia, Kubang Kerian, Kelantan, in the process of conducting this research is acknowledged.

\section{Conflict of Interest}

No conflicts of interest exist.

\section{Acknowledgment}

Guidance of Associate Professor Dr. Wan Muhamad Amir Wan Ahmad in biostatistics and assistance of Mr. Mohd Yusof Soon Abdullah in laboratory procedures is highly appreciated.

\section{References}

1 Plotino G, Grande NM, Isufi A, et al. Fracture strength of endodontically treated teeth with different access cavity designs. J Endod 2017;43(6):995-1000

2 Kishen A. Mechanisms and risk factors for fracture predilection in endodontically treated teeth. Endod Topics 2006;13:57-83

3 Tang W, Wu Y, Smales RJ. Identifying and reducing risks for potential fractures in endodontically treated teeth. J Endod 2010;36(4):609-617

4 Clark D, Khademi J. Modern molar endodontic access and directed dentin conservation. Dent Clin North Am 2010;54(2):249-273

5 Lin $\mathrm{C}-\mathrm{Y}$, Lin D, He W-H. Impacts of 3 different endodontic access cavity designs on dentin removal and point of entry in 3-dimensional digital models. J Endod 2020;46(4):524-530

6 Guler MS. Effect of access cavity designs on stress distribution. Emerg Mat Res 2020;9:220-225
7 Corsentino G, Pedullà E, Castelli L, et al. Influence of access cavity preparation and remaining tooth substance on fracture strength of endodontically treated teeth. J Endod 2018;44(9):1416-1421

8 Jain S, Kumar A, Kumar M, Kumar A. Trends in access cavity preparation: a review. Eur J Pharm Med Res 2019;6:264-268

9 Chlup Z, Žižka R, Kania J, Přibyl M. Fracture behaviour of teeth with conventional and mini-invasive access cavity designs. J Eur Ceram Soc 2017;37:4423-4429

10 Krishan R, Paqué F, Ossareh A, Kishen A, Dao T, Friedman S. Impacts of conservative endodontic cavity on root canal instrumentation efficacy and resistance to fracture assessed in incisors, premolars, and molars. J Endod 2014;40(8):1160-1166

11 Maqbool M, Noorani TY, Asif JA, Makandar SD, Jamayet N. Controversies in endodontic access cavity design: a literature review. Dent Update 2020;47:747-754

12 Neelakantan P, Khan K, Hei Ng GP, Yip CY, Zhang C, Pan Cheung GS. Does the orifice-directed dentin conservation access design debride pulp chamber and mesial root canal systems of mandibular molars similar to a traditional access design? J Endod 2018;44(2):274-279

13 Lin GSS, Ghani NRA, Noorani TY, Ismail NH. Fracture resistance of the permanent restorations for endodontically treated premolars. Eur J Gen Dent 2018;7:56-60

14 Özyürek T, Ülker Ö, Demiryürek EÖ, Yılmaz F. The effects of endodontic access cavity preparation design on the fracture strength of endodontically treated teeth: traditional versus conservative preparation. J Endod 2018;44(5):800-805

15 Abou-Elnaga MY, Alkhawas MA, Kim H-C, Refai AS. Effect of truss access and artificial truss restoration on the fracture resistance of endodontically treated mandibular first molars. J Endod 2019;45(6):813-817

16 Auswin MK, Ramesh S. Truss access new conservative approach on access opening of a lower molar: a case report. J Adv Pharm Educ Res 2017;7:345-348

17 Mooktiar H, Hedge V, Srilatha S, Chopra M. Conservative endodontics: a truss access case series. Int J Appl Dent Sci 2019;5:213-218

18 Rivera E, Walton R. Cracking the cracked tooth code: detection and treatment of various longitudinal tooth fractures. American Association Endodontists. 2019. Accessed April 29, 2021 at: https://www.aae.org/specialty/wp-content/uploads/ sites/2/2017/07/ecfesum08.pdf

19 Taha NA, Palamara JE, Messer HH. Fracture strength and fracture patterns of root filled teeth restored with direct resin restorations. J Dent 2011;39(8):527-535

20 Saberi EA, Pirhaji A, Zabetiyan F. Effects of endodontic access cavity design and thermocycling on fracture strength of endodontically treated teeth. Clin Cosmet Investig Dent 2020;12:149-156

21 Ahmed HMA. Thoughts on conventional and modern access cavity preparation techniques. Endo 2015;9:287-288 